\title{
When the skin meets the bowel: About a case*
}

\author{
Ganzetti Giulia $^{1 \#}$, Campanati Anna ${ }^{1}$, Di Sario Antonio ${ }^{2}$, Rubini Corrado ${ }^{3}$, Zizzi Antonio ${ }^{3}$, \\ Tarantino Giuseppe ${ }^{2}$, Benedetti Antonio ${ }^{2}$, Offidani Annamaria ${ }^{1}$ \\ ${ }^{1}$ Dermatological Clinic, Department of Clinical and Molecular Medicine, Polytechnic Marche University, Ancona, Italy \\ ${ }^{2}$ Gastroenterologic Clinic, Department of Clinical and Molecular Medicine, Polytechnic Marche University, Ancona, Italy \\ ${ }^{3}$ Anatomic Pathology Institute, Department of Neurosciences, Polytechnic University of Marche, Ancona, Italy \\ Email: "giulia.ganzetti@alice.it
}

Received 23 July 2013; revised 28 August 2013; accepted 15 September 2013

Copyright (c) 2013 Ganzetti Giulia et al. This is an open access article distributed under the Creative Commons Attribution License, which permits unrestricted use, distribution, and reproduction in any medium, provided the original work is properly cited.

\begin{abstract}
Ulcerative colitis (UC) is a chronic relapsing inflammatory disease of the colorectal region which may be complicated by extraintestinal manifestations, as cutaneous involvement. Immune dysregulation resulting in a lymphocyte mediated destructive process has been suggested as a possible pathogenetic link between gut and skin-related manifestations. The authors describe an atypical clinical aspect of lichen ruber planus in a patient affected by vitiligo, alopecia areata and ulcerative colitis.
\end{abstract}

Keywords: Ulcerative Colitis; Vitiligo; Lichen Ruber Planus; Alopecia Areata; Autoimmunity

\section{INTRODUCTION}

Ulcerative colitis (UC) is a chronic relapsing inflammatory disease of the colorectal region still remains to be the unknown etiology [1].

Up to $40 \%$ of IBD patients may be complicated by extraintestinal manifestations and their prevalence appears higher in Crohn disease than ulcerative colitis. Among the organ systems involved, the skin is one of the most commonly affected: up to a third of IBD patients develops cutaneous manifestations, as erythema nodosum, pyoderma gangrenosum, aphthous stomatitis, psoriasis, vitiligo, alopecia [1,2].

Vitiligo is an acquired disease affecting $0.2 \%-1 \%$ of the worldwide population and characterized by progressive, patchy, multifocal loss of skin pigmentation, overlying hair and mucous membranes [3].

Lichen planus (LP) is a chronic inflammatory eruption of the skin, scalp, nails, and mucous membranes with a reported prevalence rates of $0.5 \%-2.2 \%$. Its classic pre-

*Authors declare no conflicts of interest.

*Corresponding author. sentation is characterized by 4 p's, purple, polygonal, pruritic papules [4,5].

Alopecia Areata (AA) is a chronic inflammatory hair disease resulting in a Th1-mediated non-scarring hair loss $[2,11]$.

The authors describe the first case of concomitant lichen ruber planus, vitiligo and alopecia areata in a patient affected by ulcerative colitis.

\section{CASE REPORT}

A 50-year-old male patient was referred to our Dermatological Department for the clinical evaluation of multiple non itchy patches on the extensor surface of the right lower limb. Same erythematous lesions were detected on the right elbow (Figures 1(A) and (B)).

Lesions had started one year previously and progressively increased in number. Physical examination demonstrated erythematous, finely scalying psoriasiform eruption, localized on a vitiligo patch.

Patient's anamnesis evidenced vitiligo since 1987, ulcerative colitis for eight years; no personal nor familiar history of skin diseases and further autoimmune diseases were reported.

Ulcerative colitis was previously treated with prednisone, beclomethasone dipropionate and metronidazole ad it was in actual clinical remission: no specific inflammatory signs were found at endoscopy and laboratory data, including CRP and faecal calprotectin, were within normal ranges.

No previous treatments were done for vitiligo and psoriasiform lesions.

A skin biopsy was performed on one skin patch showing a dermal band-like chronic inflammatory infiltrate damaging basal membrane, lymphocytic exocitosis and intraepithelial cytoid bodies. Histologic features were compatible with lichen ruber planus (Figure 2(A) and (B)). Moreover, an oral examination showed a 
fine whityish network on the left cheek mucosa compatible with oral lichen planus (Figure 3(A)).

Based on the limited skin surface involved by vitiligo and lichen ruber planus, we opted for a topical therapy with clobetasol foam $0.05 \%$ daily for one month then gradually tapered for another month with completely resolution of both skin diseases.

After six weeks, the patient returned to our attention for multiple non-scarring hair loss patched of the beard.
The pull test was positive and a video-dermatoscopic analysis identified hyperkeratotic plugs (“yellow dots”), hair follicles containing cadaverized hairs ("black dots”) and broken hairs considered as pathognomonic features of alopecia areata (Figures 3(B) and (C)).

For alopecia areata, we suggested one month topical treatment with methylprednisolone aceponate $0.1 \%$ five days per week and a chili derivative product during the week end, with partial clinical resolution of the lesions.

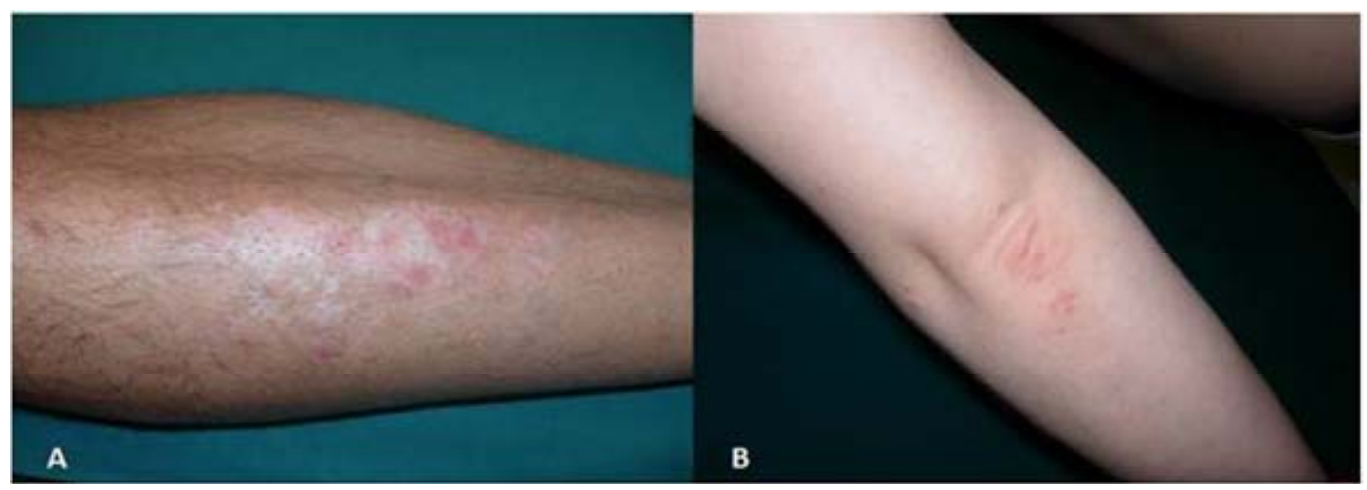

Figure 1. (A) Multiple erythematous, finely scalying patches on the extensor surface of the right lower limb localized on a vitiligo patch; (B) Erythematous, finely scalying plaque on the right elbow.
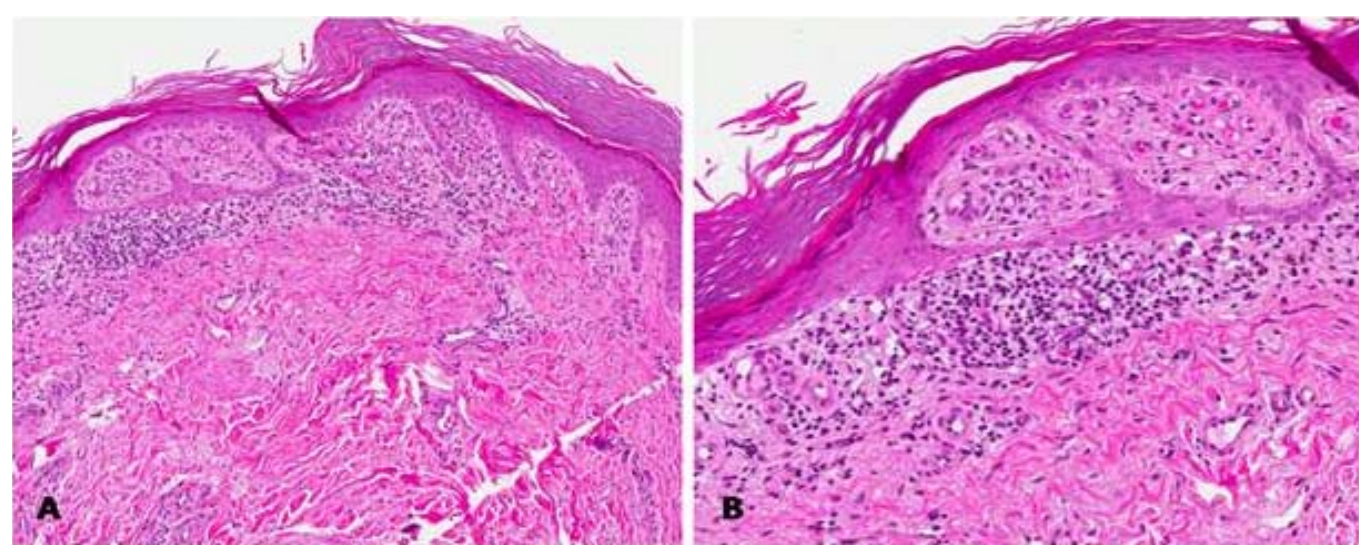

Figure 2. (A) Dermal band-like chronic inflammatory infiltrate damaging basal membrane, lymphocytic exocitosis and intraepithelial cytoid bodies; (B) A detail.

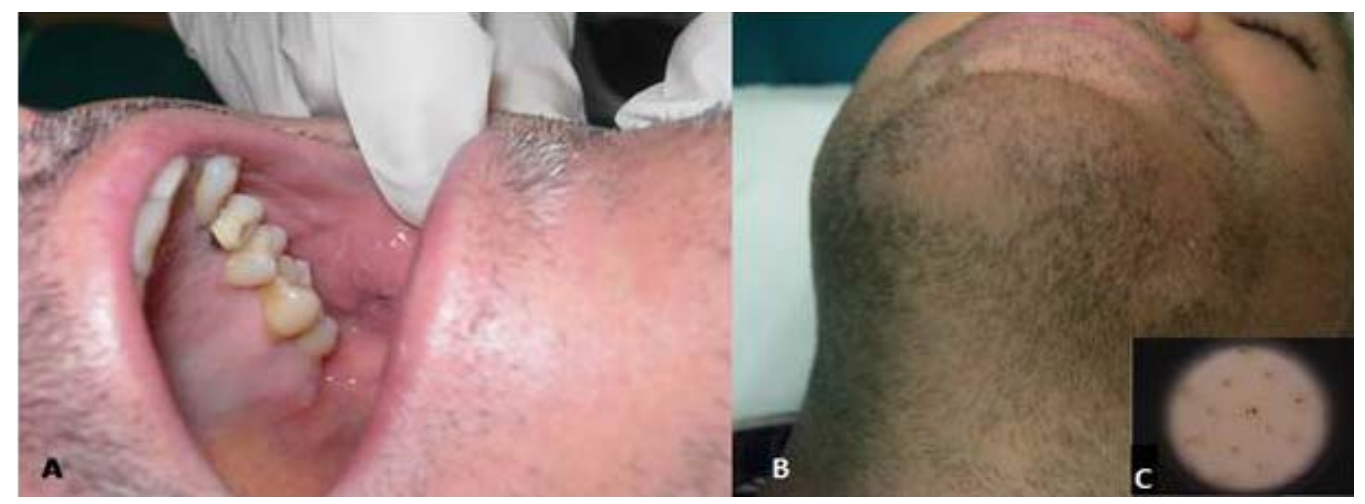

Figure 3. (A) Fine whityish network on the left cheek mucosa; (B) Multiple non-scarring hair loss patched of the beard; (C) Video-dermatoscopic findings of an alopecic patch. 


\section{DISCUSSION}

Many gastrointestinal tract disorders show cutaneous manifestations and several skin diseases may have associated to gastrointestinal lesions. Thus, the skin could mirror an occult gut disease [1,6]. Immune dysregulation resulting in a lymphocyte mediated destructive process have been suggested as a possible pathogenetic link between gut and skin-related manifestations [6].

Cytokines are important mediators of immunity and an unbalance in the cytokine network may largely determine autoimmune disease susceptibility and severity [7].

Classically, UC has been associated with Th2 mediated pathways with elevated levels of IL-5 and IL-13 [7].

However, recent evidence have suggested an important role of IL-23 and Th17 pathways both in IBD and vitiligo and lichen ruber planus; thus it could represent a further critical "meeting" point between the skin and the bowel [8-10].

In UC IL-23 may play important roles in controlling the differential Th1/Th17 balance [10].

In vitiligo, IL-17A seems to dramatically induce IL- $1 \beta$, IL-6, and TNF- $\alpha$ production in keratinocytes and fibroblasts providing evidence of the influence of a complex Th17 cell-related cytokine environment in local depigmentation in addition to CD8(+) cell-mediated melanocyte destruction [9].

In oral lichen planus, the proportion of local and peripheral Th1 and Th17 cells appears significantly increased and they may be associated with its pathogenesis [8].

Furthermore, alopecia areata is characterized by increased serum levels of IL-2, IFN- $\gamma$, IL-13, IL-17A and it emphasizes an altered T helper (Th1, Th2 and Th17) cell function and a reduced serum TGF- $\beta 1$ levels suggested defect in Tregs function [11].

In our patient, vitiligo preceded ulcerative colitis, lichen planus and alopecia areata: it could be speculated that an initial inflammatory status may have become persistent leading the compartimentalization of inflammatory processes to the gut, the skin and oral mucosa. As previously suggested, this mechanism may be linked to the homing and the trafficking of immune cells [12].

In the last 15 years, the advent of biologic therapies, particularly anti-TNF $\alpha$ antibodies, has offered new options in the management of some inflammatory diseases, such as inflammatory bowel disease and psoriasis [13].

In vitiligo, the anti-TNF-alpha agents are well tolerated but efficacy was not observed; moreover, data lack on their use in cutaneous lichen planus and a recent review have emphasized there would not seem support and rationale for use of biologics in oral lichen planus $[14,15]$.

It has been demonstrated that anti-IL12/23 antibody therapy is a highly effective for the treatment of anti TNF-alpha antibody-induced psoriasiform skin lesion in patients with inflammatory bowel disease [13].

Data are missing on the effect of anti IL-12/IL-23 treatment in vitiligo and lichen planus; although further studies are needed on this topic, it could open new perspectives on treatment of skin involvement in inflammatory bowel diseases sharing common pathogenic process.

Based on literature findings and on the actual clinical remission of ulcerative colitis, in our patient we opted for a topical steroid therapy of both vitiligo and lichen planus with satisfactory results.

The peculiarity of our patient lies in the particular clinical aspect of lichen ruber planus; in fact lesions were not the typical pruritic purple papules but erythematous finely scaling patches localized in non typical sites of lichen ruber planus.

Moreover, our reported case shows that the enhanced $\mathrm{T}$ cell mediated immunity and the breakdown of immune tolerance due to deficiency in $\mathrm{T}$ regs may facilitate the occurrence multiple autoimmune diseases creating a sort of autoimmune mosaicism.

\section{CONCLUSION}

In conclusion, identifying and accurately characterizing possible cutaneous stigmata of gastrointestinal tract disorders represent an important step in the multidisciplinary approach of patients affected by inflammatory bowel diseases.

\section{REFERENCES}

[1] Huang, B.L., Chandra, S. and Shih, D.Q. (2012) Skin manifestations of inflammatory bowel disease. Frontiers in Physiology, 3, 1-13.

http://dx.doi.org/10.3389/fphys.2012.00013

[2] Ganzetti, G., Campanati, A. and Offidani, A. (2012) Alopecia areata: A possible extraintestinal manifestation of Crohn's disease. Journal of Crohn's and Colitis, 6, 962963. http://dx.doi.org/10.1016/j.crohns.2012.05.023

[3] Taieb, A. and Picardo, M. (2009) Vitiligo. New England Journal of Medicine, 360, 160-169. http://dx.doi.org/10.1056/NEJMcp0804388

[4] Lavanya, N., Jayanthi, P., Rao, U.K. and Ranganathan, K. (2011) Oral lichen planus: An update on pathogenesis and treatment. Journal of Oral and Maxillofacial Pathology, 15, 127-132.

http://dx.doi.org/10.4103/0973-029X.84474

[5] Campanati, A., Brandozzi, G., Giangiacomi, M., Simonetti, O., Marconi, B. and Offidani, A.M. (2008) Lichen striatus in adults and pimecrolimus: Open, off-label clinical study. International Journal of Dermatology, 47, 732-736.

http://dx.doi.org/10.1111/j.1365-4632.2008.03654.x

[6] Ghevariya, V., Singhal, S. and Anand, S. (2013) The skin: A mirror to the gut. International Journal of Colorectal Disease, 28, 889-913.

http://dx.doi.org/10.1007/s00384-012-1637-x 
[7] Van Wijk, F. and Cheroutre H. (2010) Mucosal T cells in gut homeostasis and inflammation. Expert Review of Clinical Immunology, 6, 559-566. http://dx.doi.org/10.1586/eci.10.34

[8] Xie, S., Ding, L., Xiong, Z. and Zhu, S. (2012) Implications of Th1 and Th17 cells in pathogenesis of oral lichen planus. Journal of Huazhong University of Science and Technology-Medical Sciences, 32, 451-457. http://dx.doi.org/10.1007/s11596-012-0078-7

[9] Kotobuki, Y., Tanemura, A., Yang, L., Itoi, S., et al. (2012) Dysregulation of melanocyte function by Th17related cytokines: Significance of Th17 cell infiltration in autoimmune vitiligo vulgaris. Pigment Cell \& Melanoma Research, 25, 219-230. http://dx.doi.org/10.1111/j.1755-148X.2011.00945.x

[10] Kobayashi, T., Okamoto, S., Hisamatsu, T., et al. (2008) IL23 differentially regulates the Th1/Th17 balance in ulcerative colitis and Crohn's disease. Gut, 57, 1682-1689. http://dx.doi.org/10.1136/gut.2007.135053

[11] Tembhre, M.K. and Sharma, V.K. (2013) T helper and regulatory $\mathrm{T}$ cell cytokines in the peripheral blood of ac- tive alopecia areata. British Journal of Dermatology, $\mathbf{1 6 9}$, 543-548. http://dx.doi.org/10.1111/bjd.12396

[12] Mann, E.R., McCarthy, N.E., Peake, S.T.C., et al. (2012) Skin- and gut-homing molecules on human circulating $\gamma \delta$ $\mathrm{T}$ cells and their dysregulation in inflammatory bowel disease. Clinical \& Experimental Immunology, 170, 122 130. http://dx.doi.org/10.1111/j.1365-2249.2012.04649.x

[13] Tillack, C., Ehmann, L.M., Friedrich, M., et al. (2013) Anti-TNF antibody-induced psoriasiform skin lesions in patients with inflammatory bowel disease are characterised by interferon- $\gamma$-expressing Th1 cells and IL-17A/IL22-expressing Th17 cells and respond to anti-IL-12/IL-23 antibody treatment. Gut. http://www.ncbi.nlm.nih.gov/pubmed/23468464

[14] Alghamdi, K.M., Khurrum, H., Taieb, A. and Ezzedine, K. (2012) Treatment of generalized vitiligo with antiTNF- $\alpha$ Agents. Journal of Drugs in Dermatology, 11, 534-539.

[15] O’Neill, I.D. and Scully, C. (2013) Biologics in oral medicine: Ulcerative disorders. Oral Diseases, 19, 37-45. http://dx.doi.org/10.1111/j.1601-0825.2012.01931.x 